\title{
A Hybrid Method for Two-dimensional Sound-soft Crack Reconstruction
}

\author{
Rainer Kress ${ }^{1}$ and Pedro Serranho ${ }^{2}$
}

\begin{abstract}
We are interested in solving the inverse problem of acoustic wave scattering by sound soft cracks in two-dimensions. Therefore, we present a method to approximate the position and shape of a two-dimensional sound-soft crack from a given incident field and the corresponding far-field pattern of the scattered wave. The method is based on a recent paper by Chapko and Kress, where a hybrid method is presented for the reconstruction of sound-soft domains. The method can be seen has a hybrid between a regularized Newton method applied to a nonlinear operator equation, where we consider the operator that maps the unknown curve (representing the crack) on the solution of the direct problem, and a decomposition method, in the spirit of the Kirsch and Kress method. As well as in the method developed by Chapko and Kress, the method does not require a forward solver for each Newton step. The theoretical background of the method is based on the minimization of a cost function, where we add an additional penalty term in order to recover the crack tips. The method is illustrated by numerical examples, showing its feasibility and stability with respect to noisy data.
\end{abstract}

\section{Introduction}

Non destructive obstacle detecting trough low frequency wave propagation originates mathematical and numerical problems with several applications in industry, as radar and sonar or medical imaging. Among this problems, we are interested in numerical methods for recovering cracks in materials from the knowledge of the incident field and the scattered field at large distances (far field pattern). We confine ourselves to the case of time harmonic acoustic waves and the scattering from sound soft cracks.

Given a crack $\Gamma \subset B \subset \mathbb{R}^{2}$, with $B$ an open bounded domain, and given an incident field $u^{i}$, the direct problem consists in finding the total field $u=u^{i}+u^{s c}$, which is the sum of the incident field $u^{i}$ and the scattered field $u^{s c}$, such that both the Helmholtz equation

$$
\Delta u+k^{2} u=0 \quad \text { in } \mathbb{R}^{2} \backslash \Gamma
$$

where $k>0$ is the wave number, and the boundary Dirichlet condition

$$
u=0 \quad \text { on } \Gamma
$$

\footnotetext{
${ }^{1}$ Institut für Numerische und Angewandte Mathematik, Georg-August Universität, 37083 Göttingen, Germany, (kress@math.uni-goettingen.de)

${ }^{2}$ Institut für Numerische und Angewandte Mathematik, Georg-August Universität, 37083 Göttingen, Germany, (serranho@math.uni-goettingen.de)
} 
are satisfied. Concerning well-posedness, one also needs to impose a condition to infinity. We consider the Sommerfeld radiation condition

$$
\lim _{r \rightarrow \infty} \sqrt{r}\left(\frac{\partial u}{\partial r}-i k u\right)=0, \quad r=|x|
$$

where the limit is satisfied uniformly in all directions. It is known (cf. [2]) that the solution $u$ satisfies

$$
u(x)=\frac{e^{i k|x|}}{\sqrt{|x|}}\left(u_{\infty}(\hat{x})+O\left(\frac{1}{|x|}\right)\right), \quad|x| \rightarrow \infty,
$$

where $\hat{x}=x /|x|$, so the behaviour of the solution $u$ to infinity is completely determined by the far field pattern $u_{\infty}$.

Among all the methods that were developed to solve the problem, we would like to focus on regularized Newton's methods and decomposition methods.

For a fixed incident field, the solution of the direct problem defines the operator

$$
F: \Gamma \mapsto u_{\infty}
$$

that maps the crack $\Gamma$ onto the far field. We can formulate the inverse problem as finding the solution of the nonlinear and ill-posed (cf. [2]) operator equation

$$
F(\Gamma)=u_{\infty}
$$

for the unknown crack $\Gamma$.

Regularized Newton's methods have been studied and used in this problem over two decades. It is reasonable to apply them because one loses the nonlinearity and, by regularization, the ill-posedness. These methods rely on the differentiability of the total field or far field pattern in relation to variations of the scatterer. The reconstructions obtained by this methods are usually very accurate, however for each step of the Newton's method one needs to compute the forward solution of the direct problem. A good a priori initial approximation is usually also required.

Decomposition methods take care of the ill-posedness and the nonlinearity of the equation separately. In a first step the function $u$ is reconstructed given the far field, by representing it in a single layer potential over a line $\gamma$, which shall be viewed as an approximation of the crack $\Gamma$. In this way we reconstruct the density over $\gamma$ using Tikhonov regularization. Then in a second step we try to find a line where the boundary condition (2) is satisfied in a minimization sense. Though this method does not need the solution of the forward problem, the reconstructions obtained are not highly accurate. There is also a gap between the theoretical background and the numerical implementation, in what concerns uniqueness and existence of solution of the minimization problem involved.

Chapko and Kress [1] have suggested a hybrid method that combines ideas of the two groups of numerical methods previously mentioned. In this paper we extend the method to the case of sound soft cracks. 
This paper is organized as follows. In section 2 is given an overview of the direct problem of scattering by cracks, pointing out what are the main differences to scattering by open domais. In section 3 we formulate the inverse problem we want to solve - to find the position and shape of a two-dimensional sound soft crack given an incident field and the corresponding far field - while in section 4 is made an overview of the numerical implementation. A related minimization problem is presented in section 5 as the theoretical background for the method, where a cost function is introduced with a new penalty term to allow better crack tips reconstruction. Finally, we present numerical results which show the feasibility of the method, even with noisy data.

\section{Direct Problem}

In order to solve the direct problem introduced in the previous section, we use a single layer representation of the solution. We introduce the single layer operator $S_{z}: L^{2}\left(\Gamma_{z}\right) \rightarrow L^{2}\left(\mathbb{R}^{2}\right)$ given by

$$
\left(S_{z} \varphi\right)(x)=\int_{\Gamma_{z}} \Phi(x, y) \varphi(y) d S y, \quad x \in \mathbb{R}^{2},
$$

where $\varphi \in L^{2}(\Gamma)$ is a density and the fundamental solution $\Phi$ is given by

$$
\Phi(x, y)=\frac{i}{4} H_{0}^{(1)}(k|x-y|), \quad x \neq y,
$$

in terms of the Hankel function $H_{0}^{(1)}$ of the first kind and order zero. We can now represent the solution $u$ as

$$
u(x)=\left(S_{z} \varphi\right)(x)+u^{i}(x), \quad x \in \mathbb{R}^{2} .
$$

By the boundary condition (2) we want to solve the integral equation

$$
\left(S_{z} \varphi\right)(x)=-u^{i}(x) \quad \text { on } \Gamma
$$

in order to the unknown density $\varphi$. The density $\varphi$ is assumed to have the form

$$
\varphi(x)=\frac{\tilde{\varphi}(x)}{\left|x-z_{1}\right|\left|x-z_{-1}\right|}, \quad x \in \Gamma_{z} \backslash\left\{z_{-1}\right\} \cup\left\{z_{1}\right\}
$$

where $\tilde{\varphi}$ is a bounded function and $z_{-1}, z_{1}$ are the crack tips. Therefore $\varphi$ might have singularities at the endpoints of the crack $\Gamma$. In order to lose these singularities one uses the cosinus substitution (cf. Yan, Sloan [8]) and introduces the operator $\tilde{S}_{z}: L^{2}[0,2 \pi] \rightarrow L^{2}[0,2 \pi]$ given by

$$
\left(\tilde{S}_{z} \psi\right)(t)=\int_{0}^{2 \pi} H(t, \tau) \psi(\tau) d \tau, \quad t \in[0,2 \pi] .
$$

where $\psi \in L^{2}[0,2 \pi]$ is now a bounded $2 \pi$-periodic even function (cf. Kress [5]) given by

$$
\psi(t)=\varphi(z(\cos t))\left|z^{\prime}(\cos t)\right||\sin t|
$$


and

$$
H(t, \tau)=\frac{i}{8} H_{0}^{(1)}(k|z(\cos t)-z(\cos \tau)|), \quad t \neq \tau .
$$

From (5) one wants to solve the equation

$$
\left(\tilde{S}_{z} \psi\right)(t)=-u^{i}(z(\cos (t))) \quad t \in[0,2 \pi]
$$

in order to $\psi$. As the kernel $H(t, \tau)$ has a logarithmic singularity at $t=\tau$ we need to use a proper integration rule. We use the same proceeding as in [5], that is, we consider trignometric interpolation of the density $\psi$ and use an exact quadrature rule on the trignometric interpolation polynomial which considers explicitly the logarithmic singularity of the kernel.

Afterwards, one can approximate the solution of the problem using

$$
u(x)=\frac{i}{4} \int_{0}^{\pi} H_{0}^{(1)}(k|x-z(\cos \tau)|) \psi(\tau) d \tau+u^{i}(x), \quad x \in \mathbb{R}^{2} .
$$

Introducing the far field operator $F_{z}: L^{2}\left(\Gamma_{z}\right) \rightarrow L^{2}(\Omega)$, where

$$
\Omega=\left\{x: x \in \mathbb{R}^{2},|x|=1\right\},
$$

given by

$$
\left(F_{z} \varphi\right)(\hat{x})=\frac{e^{i \frac{\pi}{4}}}{\sqrt{8 \pi k}} \int_{\Gamma_{z}} e^{-i k \hat{x} \cdot y} \varphi(y) d S y, \quad \hat{x} \in \Omega
$$

and using once more the cosinus subtitution, one introduces the new operator $\tilde{F}_{z}: L^{2}([0,2 \pi]) \rightarrow L^{2}(\Omega)$ given by

$$
\left(\tilde{F}_{z} \psi\right)(\hat{x})=\frac{e^{i \frac{\pi}{4}}}{\sqrt{8 \pi k}} \int_{0}^{\pi} e^{-i k \hat{x} . z(\cos \tau)} \psi(\tau) d \tau, \quad \hat{x} \in \Omega .
$$

One can now represent the far field by

$$
u_{\infty}(\hat{x})=\left(\tilde{F}_{z} \psi\right)(\hat{x}), \quad \hat{x} \in \Omega
$$

where $\psi$ is the solution of (7).

We are interested in solving the inverse problem related to the previous direct problem, i.e., given $u^{i}$ and the far field pattern $u_{\infty}$, we want to determine the position and shape of the crack $\Gamma$, knowing that (1), (2) and (3) are satisfied.

\section{Inverse Problem}

Given the far field pattern $g$, which may be perturbed, we want to approximate the position and shape of the crack. We introduce the operator $G$ defined by

$$
G:\left.\gamma \mapsto u\right|_{\gamma}
$$

that maps a line $\gamma \in \mathbb{R}^{2}$ onto the trace of the total field $u$ on $\gamma$. 
Our goal is to find $\gamma$ such that

$$
G(\gamma)=0,
$$

according to the boundary condition (2). Considering a parametrization $z$ of $\gamma$ we can rewrite the previous equation as

$$
G(z)=0
$$

where we use the same symbol for the operator $G$, though this operator is now defined in a parametrization space. By linearizing the equation, we are led to

$$
G(z+h)=G(z)+G^{\prime}(z) h+O\left(h^{2}\right)
$$

and, as the Frechet derivative of $G$ is obviously given by

$$
G^{\prime}(z) h=\left.\operatorname{grad} u\right|_{z} \cdot h,
$$

we get the linearized equation

$$
\left.u\right|_{z}+\left.\operatorname{grad} u\right|_{z} \cdot h=0,
$$

once, given a $z$, we want to find $h$ such that $G(z+h)=0$, in a minimization sense.

Considering that $\Gamma$ is our exact solution, it is important to notice that for every line $\gamma \subset \Gamma$ we have

$$
\left.u\right|_{\gamma}=0
$$

and therefore it might be expected that the solution from (11) will lead us to a line $\gamma \subset \Gamma$ that is not the crack itself. To prevent this we need to impose some condition on the length of the new crack $\Gamma_{z+h}$. So instead of looking for a solution of (11) we will look for $h$ that minimizes

$$
\left\|u+\operatorname{grad} u .\left.h\right|_{L^{2}\left(\Gamma_{z}\right)}-\nu\right\| \Gamma_{z+h} \|
$$

for some small constant $\nu>0$.

This is the main idea for each iteration of our method. Given an initial guess $z$, one minimizes (12) to get $h$ and establishes $z+h$ as the new guess, repeating this process iteratively.

\section{Numerical Implementation}

Let

$$
\Gamma_{z}=\{z(t): t \in[-1,1]\}
$$

be a crack defined by $z:[-1,1] \rightarrow \mathbb{R}^{2}$. We will only consider parametrizations $z$ in the bounded subset $V \subset H^{l}[-1,1]$ which are admissible. We want bounded cracks, i.e., bounded lines in $\mathbb{R}^{2}$, which do not intersect themselves. Therefore we need the following definition of admissible cracks. 
Definition 1. Let $x \in \Gamma_{z}$ and $t_{x} \in[0, \pi]$ such that $x=z\left(\cos t_{x}\right)$. A parametrization $z$ is called $\varepsilon-$ admissible if for every $x \in \Gamma_{z}$ and every $0<\eta<\varepsilon$ there exists $t_{1}, t_{2} \in[0, \pi], t_{1}<t_{x}<t_{2}$, such that

$$
\Gamma_{z} \cap B(x, \eta)=\left\{z(\cos t): t_{1}<t<t_{2}\right\} .
$$

It is easy to show that for each $\varepsilon>0$ the space of $\varepsilon$-admissible parametrizations is closed.

We consider an initial crack $\Gamma_{z}$ and according to the previous section we want to find $h$ such that (11) is satisfied. Therefore we need to have an approximation of the total field and its gradient on $\Gamma_{z}$. Given the far field $g$, one starts by solving the equation

$$
\left(\tilde{F}_{z} \psi\right)(\hat{x})=g(\hat{x}), \quad \hat{x} \in \Omega
$$

in order to $\psi$ by applying the Nyström method to the Tikhonov regularized equation.

Then we can now approximate the total field $u$ by the single layer representation (8). To approximate the gradient we use the decomposition in the normal and tangential derivative of the total field $u$, that is,

$$
\operatorname{grad} u(x)=\frac{\partial u}{\partial s} s_{x}+\frac{\partial u}{\partial n} n_{x}
$$

where $s_{x}$ and $n_{x}$ are the tangential and normal unit vectors to the crack on its point $x$. For the tangential derivative we use trignometric differentiation of $u(z(\cos t))$ for $t \in[0, \pi]$, that is, we use the derivative of the trignometric interpolation polynomial of the previous function on the nodes considered in the Nyström method as approximation of the tangential derivative of the total field, multiplying it by the jacobian of the variable transformation. Concerning the normal derivative, one knows that

$$
\frac{\partial u_{ \pm}}{\partial n}(x)=\mp \frac{\varphi(x)}{2}+\frac{i}{4} \int_{0}^{\pi} \frac{\partial}{\partial n} H_{0}^{(1)}(k|x-z(\cos \tau)|) \psi(\tau) d \tau+\frac{\partial u^{i}}{\partial n}(x), \quad x \in \Gamma_{z},
$$

where, by definition,

$$
\left.\varphi(z(\cos t))=\frac{\psi(t)}{\left|z^{\prime}(\cos (t))\right||\sin (t)|}, t \in\right] 0, \pi[.
$$

Once more one needs to be careful with the quadrature rules used because both kernels involved have singularities. For details we refer to [2].

Considering a finite n-dimensional parametrization subspace $V_{n} \subset V$, we want to minimize (12) in order to the parametrization $h$. The minimization is made by a Levenberg- Marquardt step.

Remark 1. At each point considered in the minimization approximation, one needs to decide which value of the gradient of the total field to choose. As we want $u$ to be zero, we choose the side in which the normal derivative of the absolute value of the total field has the steepest descent, according to the quantity

$$
\frac{\partial}{\partial n}\left|u_{ \pm}\right|^{2}=2 \operatorname{Re}\left(\bar{u} \frac{\partial u_{ \pm}}{\partial n}\right)
$$


Remark 2. At the endpoints of the crack the normal derivative might have singularities according to (14). Therefore, once the Newton method uses the gradient of $u$ to move the current approximation to the next one, it might me expected that the successive approximations do not move in the normal direction near the crack tips. To prevent this problem, we introduce a weight in the minimization with decay

$$
\sqrt{\left(x-z_{-1}\right)\left(x-z_{1}\right)}
$$

as $x \in \Gamma_{z_{n}}$ goes to the endpoints $z_{-1}$ and $z_{1}$. However, this may bring less accuracy at the crack tips reconstruction.

\section{Minimization Problem}

In this section we will consider a minimization problem that is related with the hybrid method presented in the previous sections.

We introduce the cost function $\mu(., ., \alpha, \nu): L^{2}[0,2 \pi] \times V \rightarrow \mathbb{R}$ defined by

$$
\mu(\psi, z, \alpha, \nu):=\alpha\|\psi\|_{L^{2}[0,2 \pi]}^{2}-\nu\left\|\Gamma_{z}\right\|^{2}+\mu_{1}(\psi, z)+\mu_{2}(\psi, z)
$$

where

$$
\mu_{1}(\psi, z):=\left\|\tilde{F}_{z}(\psi)-u_{\infty}\right\|_{L^{2}(\Omega)}^{2}
$$

and

$$
\mu_{2}(\psi, z):=\left\|S_{z}\left(\psi \circ \arccos \circ z^{-1}\right)+u^{i n c}\right\|_{L^{2}\left(\Gamma_{z}\right)}^{2} .
$$

Our goal is to minimize $\mu$ in order to $z$ and $\psi$, given some fixed small $\alpha$ and $\nu$. We introduce the following definition.

Definition 2. A crack $\Gamma_{z}$ is called optimal if there exists a function $\psi \in L^{2}[0,2 \pi]$ such that the pair $(\psi, z)$ minimizes the cost function $\mu$ over $L^{2}[0,2 \pi] \times V$.

We can now establish the following results, which are related with the existence and uniqueness of the minimum of the cost function $\mu$.

Theorem 1. Assume $l>5 / 2$. Then for each $g \in L^{2}(\Omega)$ and each regularization parameters $\alpha$ and $\nu$ there exists an optimal curve.

Proof. We will follow Chapko, Kress [1]. Consider $\left(\psi_{n}, z_{n}\right)$ a minimization sequence in $L^{2}[0,2 \pi] \times V$, i.e.,

$$
\lim _{n \rightarrow \infty} \mu\left(\psi_{n}, z_{n}, \alpha, \nu\right)=M(\alpha, \nu)
$$

where

$$
M(\alpha, \nu)=\inf _{\psi \in L^{2}[0,2 \pi], z \in V} \mu(\psi, z, \alpha, \nu) .
$$

By the Sobolev imbedding theorems, one knows that under the assumptions on $l, H^{l}[0,2 \pi] \subset C^{2}[0,2 \pi]$. As $V$ is bounded, one can assume convergence $z_{n} \rightarrow z$ as $n \rightarrow \infty$ in $C^{2}$ norm. Clearly we have that $z \in V$ and that

$$
\lim _{n \rightarrow \infty}\left\|\Gamma_{z_{n}}\right\|=\left\|\Gamma_{z}\right\| .
$$


Once $\alpha>0$ and

$$
\alpha\left\|\psi_{n}\right\|_{L^{2}[0,2 \pi]}^{2} \leq \mu\left(\psi_{n}, z_{n}, \alpha, \nu\right)+\nu\left\|\Gamma_{z_{n}}\right\| \rightarrow M(\alpha, \nu)+\nu\left\|\Gamma_{z}\right\|, \quad n \rightarrow \infty,
$$

we have that the sequence $\left(\psi_{n}\right)$ is bounded. Therefore, one can assume weak convergence $\psi_{n} \rightarrow \psi$ as $n \rightarrow \infty$.

Since both $\tilde{F}_{z}: L^{2}[0,2 \pi] \rightarrow L^{2}\left(S^{2}\right)$ and $S_{z}: L^{2}\left(\Gamma_{z}\right) \rightarrow L^{2}\left(\Gamma_{z}\right)$ are compact operators and Frechet differentiable, from the convergence $z_{n} \rightarrow z \in C^{2}$ one gets operator norm convergence (cf. Colton,Kress [2])

$$
\lim _{n \rightarrow \infty}\left\|\tilde{F}_{z}-\tilde{F}_{z_{n}}\right\|_{L^{2}(\Omega)}=0 \quad \text { and } \quad \lim _{n \rightarrow \infty}\left\|S_{z}-S_{z_{n}}\right\|_{L^{2}(\Gamma)}=0 .
$$

From the limits, using the triangle inequality and the boundedness of the sequence $\left(\psi_{n}\right)$ one gets

$$
\lim _{n \rightarrow \infty} \tilde{F}_{z_{n}}\left(\psi_{n}\right)=\tilde{F}_{z}(\psi)
$$

and

$$
\lim _{n \rightarrow \infty} S_{z_{n}}\left(\psi_{n} \circ \arccos \circ z^{-1}\right)=S_{z}\left(\psi \circ \arccos \circ z^{-1}\right) .
$$

Hence we have convergence

$$
\alpha\left\|\psi_{n}\right\|_{L^{2}[0,2 \pi]}^{2} \rightarrow M(\alpha)-\mu_{1}(\varphi, z)-\mu_{2}(\varphi, z)+\nu\left\|\Gamma_{z}\right\|^{2} \leq \alpha\|\psi\|_{L^{2}[0,2 \pi]}^{2}
$$

for $n \rightarrow \infty$. It follows from the previously established weak convergence that

$$
\lim _{n \rightarrow \infty}\left\|\psi_{n}-\psi\right\|_{L^{2}[0,2 \pi]}^{2}=\lim _{n \rightarrow \infty}\left\|\psi_{n}\right\|_{L^{2}[0,2 \pi]}^{2}-\lim _{n \rightarrow \infty}\|\psi\|_{L^{2}[0,2 \pi]}^{2} \leq 0,
$$

which means we also have norm convergence $\psi_{n} \rightarrow \psi, n \rightarrow \infty$. By continuity one can now establish the result.

Theorem 2. Assume $l>5 / 2$ and let $\left(\alpha_{n}\right)$ and $\left(\nu_{n}\right)$ be two null sequence and $\left(z_{n}\right)$ be the corresponding sequence of solutions to the optimization problem with regularization parameters $\alpha_{n}$ and $\nu_{n}$. Assume that $g$ is the exact far field pattern of the solution of the Dirichlet Problem for the crack $\Gamma_{z}$ with $z \in V$. Then, every limit point $z^{*}$ of $z_{n}$ represents a crack on which the total field vanishes.

Proof. Since we can represent the solution of (1) and (2) using a single layer potential by (8) using the solution $\psi$ of (13) for $\Gamma_{z}$, and once $g$ is the exact far field pattern one clearly gets $\mu_{1}(\psi, z)=\mu_{2}(\psi, z)=0$, which means

$$
M(\alpha, \nu)=\alpha\|\psi\|_{L^{2}[0,2 \pi]}^{2}-\nu\left\|\Gamma_{z}\right\|^{2} \rightarrow 0, \quad \alpha \rightarrow 0, \nu \rightarrow 0 .
$$

As in the previous proof one can assume that there exists a $C^{2}$ convergent subsequence $z_{n(k)} \rightarrow z^{*}, k \rightarrow \infty$, where $z^{*} \in V$. Let $u^{*}$ be the solution to the problem considering the crack $\Gamma_{z^{*}}$. As $z_{n(k)}$ is optimal for the parameters $\alpha_{n(k)}, \nu_{n(k)}$, there exists $\psi_{n(k)} \in L^{2}(0,2 \pi)$ such that

$$
\mu\left(\psi_{n(k)}, z_{n(k)}, \alpha_{n(k)}, \nu_{n(k)}\right)=M\left(\alpha_{n(k)}, \nu_{n(k)}\right), \quad k=1,2, \ldots
$$


Therefore

$$
\mu_{2}\left(\psi_{n(k)}, z_{n(k)}\right) \leq M\left(\alpha_{n(k)}, \nu_{n(k)}\right)+\nu_{n(k)}\left\|\Gamma_{z_{n(k)}}\right\| \rightarrow 0, \quad k \rightarrow \infty .
$$

From this and from the fact that $u^{*}=0$ on $\Gamma_{z^{*}}$, by the continuous dependence of the solution of the problem on the crack in the $L^{2}$ sense, we get

$$
\left\|\tilde{F}_{z_{n(k)}}(\psi)-u_{\infty}^{*}\right\|_{L^{2}\left(S^{2}\right)}^{2} \rightarrow 0, \quad k \rightarrow \infty
$$

where $u_{\infty}^{*}$ holds for the far field pattern of $u^{*}$. As we also have that

$$
\left\|\tilde{F}_{z}(\psi)-g\right\|_{L^{2}\left(S^{2}\right)}^{2} \leq M\left(\alpha_{n(k)}, \nu_{n(k)}\right)+\nu_{n(k)}\left\|\Gamma_{z_{n(k)}}\right\| \rightarrow 0, \quad k \rightarrow \infty
$$

we conclude that

$$
g=u_{\infty}^{*} \quad \text { on } S^{2}
$$

and so $u=u^{*}$ as a consequence of the Rellich Lemma. Therefore

$$
u^{*}+u^{i}=0 \quad \text { on } \Gamma,
$$

which completes the proof.

In practice, the hybrid method does not minimize globally the cost function $\mu$. The minimization is divided in two parts. One starts by minimizing the function

$$
\alpha\|\psi\|_{L^{2}(\Gamma)}+\mu_{1}(\psi, z)
$$

in order to $\psi$ for a fixed $z$, and then minimizes the function

$$
\mu_{2}(\psi, z)-\nu\left\|\Gamma_{z}\right\|
$$

in order to $z$, for $\psi$ the solution of the first minimization problem, using a Levenberg-Marquardt step. Therefore this theoretical background still needs some work.

\section{Numerical Examples}

We will now present a few numerical examples of the method presented in the previous sections.

As space of parametrizations we choose

$$
V_{N, C}=\left\{z(.)=\left(\sum_{j=0}^{N} a_{j} T_{j}(.), \sum_{j=0}^{N} b_{j} T_{j}(.)\right): a_{j}, b_{j} \in \mathbb{R},\left|a_{j}\right|<C,\left|b_{j}\right|<C\right\}
$$

where $T_{j}$ holds for the Chebyshev polynomial of degree $j$, that is, parametrizations which both components are linear combinations of Chebyshev polynomials of degree less or equal to $N$. The choice of Chebyshev polynomials is natural, since the parametrizations are defined in $[-1,1]$, and it allows to approximate a great variety of cracks. 
In the examples through this section we will consider the incident plane field given by

$$
u^{i}(x)=e^{i k x . d}
$$

where the wave number is $k=1$ and the propagation direction is $d=(0,1)$. We will use Chebyshev polynomials of degree less or equal to $\mathrm{N}=5$.

We use 40 points for reconstruct the density $\psi$ in (13) and 30 points for the minimization by the Levenberg-Marquardt step of (12). In the examples shown, the parameter $\nu$ in (12) is settled to be $\nu=10^{-2}$. Numerical experiments showed us that this choice should be made such that the minimum of the cost function is close to zero, which requires more a priori information on the length of the crack.

We start by presenting a reconstruction of a parabolic crack defined by

$$
z(t)=\left(t, t^{2}\right)
$$

in figure 1. The approximation is very good, even with a perturbed far field pattern.

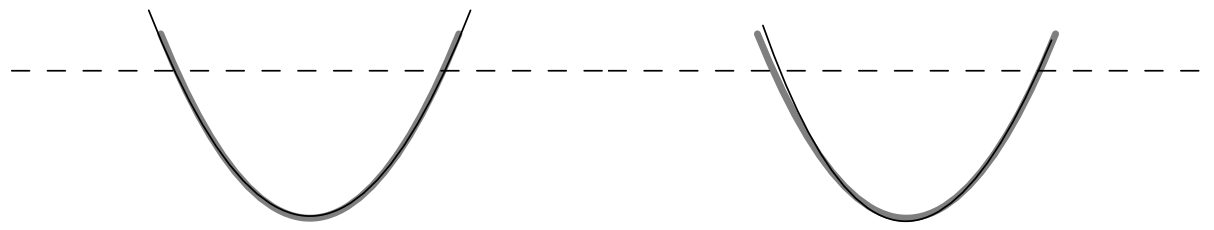

Figure 1: Comparison of the approximation (in black) obtain with the given initial guess (dashed line) with no noise (left) and $5 \%$ noise (right) and the exact crack (in grey).

In figure 2 we present a reconstruction of a crack that can not be given by a graph of a function, with parametrization

$$
z(t)=\left(t+t^{2}, t+t^{4} / 2\right) .
$$

We note that in this case, due to the extreme curvature near one of the crack points, the quality of the approximation depends greatly on the initial guess given. The reconstruction presented is also very good, both in shape and position of the crack.

As last example, we present a reconstruction of a crack that is out of the approximation space $V_{N, C}$, with parametrization

$$
z(t)=(t, \sin (1.5 t)) .
$$

The results are presented in figure 3 . This example shows also what was pointed out in remark 2 , as the reconstruction in the middle of the crack is much better than in its endpoints. 

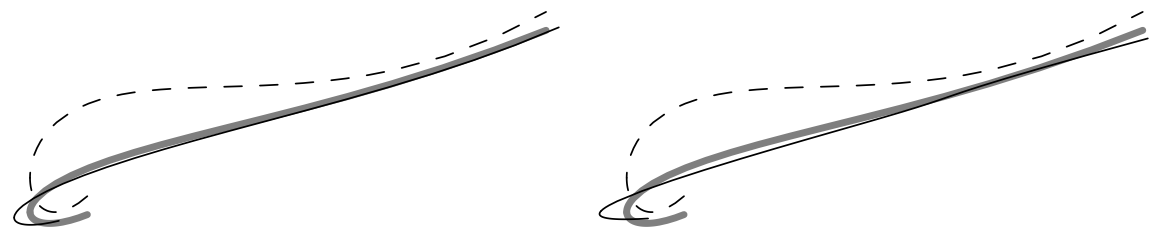

Figure 2: Comparison of the approximation (in black) obtain with the given initial guess (dashed line) with no noise (left) and $5 \%$ noise (right) and the exact crack (in grey).

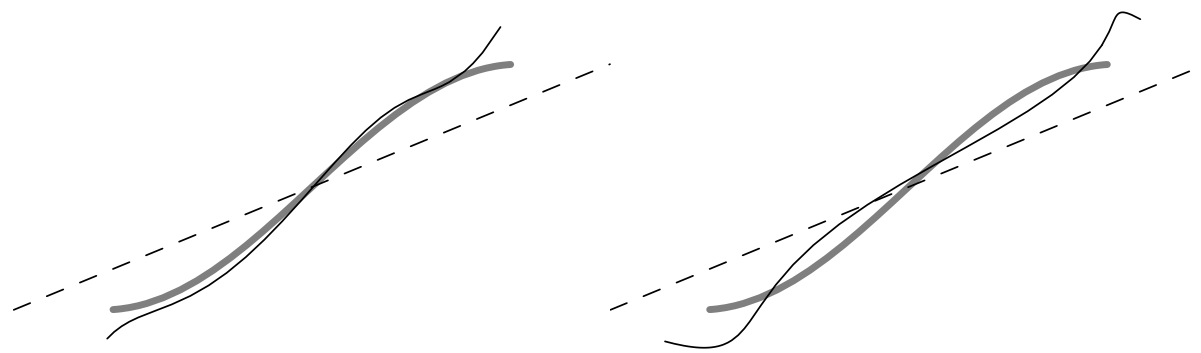

Figure 3: Comparison of the approximation (in black) obtain with the given initial guess (dashed line) with no noise (left) and $5 \%$ noise (right) and the exact crack (in grey) .

The results show that the method gives accurate reconstructions of the crack, even with noisy data, though a good choice of an initial guess is needed. However the approximation of the crack tips still needs improvement, once, in some cases, it is not so accurate as in the middle part of the crack.

\section{Acknowledgments}

The second author would like to acknowledge that Fundação para a Ciência e a Tecnologia supports his work through the referenced SFRH/BD/14248/2003 scholarship.

\section{References}

[1] Chapko R., Kress R., A hybrid method for inverse boundary values problems in potential theory, (to appear)

[2] Colton D., Kress R., Inverse Acoustic and Electromagnetic Scattering Theory, $2^{\text {nd }}$ Edition, Springer, 1998

[3] Hohage T., Convergence rates of a regularized Newton method in soundhard inverse scattering, SIAM J. Numer. Anal. 36, 125,142, 1998 
[4] Kress R., Linear Integral Equations, $2^{\text {nd }}$ Edition, Springer, 1999

[5] Kress R., Inverse Scattering from an Open Arc, Math. Met. in Appl. Sciences 18,1995

[6] Kress R., Rundell W., Inverse Scattering for shape and impedance, Inverse Problems 17, 1075-1085, 2001

[7] Potthast R., On the convergence of a new Newton-type method in inverse scattering, Inverse Problems 17, 1419-1434, 2001

[8] Yan Y., Sloan I.H., On the Integral equation of the first kind with logarithmic kernels, J. Integral equations Appl. 1, 549-579, 1988 